\title{
DESA: Dependable, Efficient, Scalable Architecture for Management of Large-Scale Batteries
}

\author{
Hahnsang Kim, Member, IEEE, and Kang G. Shin, Fellow, IEEE
}

(Invited Paper)

\begin{abstract}
Conventional battery management systems (BMSs) for electric vehicles (EVs) are designed in an ad hoc way, causing the supply of EVs to fall behind the market demand. A well-designed and combined hardware-software architecture is essential for the efficient management of a large-scale battery pack that may consists of thousands of battery cells as in Tesla Motors and GM Chevy Volt. We propose a Dependable, Efficient, Scalable Architecture (DESA) that effectively monitors a large number of battery cells, efficiently controls, and reconfigures, if needed, their connection arrangement. DESA supports hierarchical, autonomous management of battery cells, where a global BMS orchestrates a group of local BMSs. A local controller on each local BMS autonomously manages an array of battery cells, and the global controller reconfigures the connectivity of such battery-cell arrays in coordination with the local controllers. Also, DESA allows individual arrays and local BMSs to be selectively powered-off for energy savings. The performance of this energy-saving capability is modeled and evaluated using a Markov chain. Our evaluation results show that DESA effectively tolerates battery-cell failures by an order-of-magnitude-while achieving $7.4 \times$ service cost savings-better than a conventional BMS. This superior performance not only extends the battery life significantly, but also provides the flexibility in supporting diverse electric power demands from a growing number of on-board applications.
\end{abstract}

Index Terms-Battery cells and packs, battery management system (BMS), electric vehicles, reconfiguration of cell and pack connections, voltage and cell balancing.

\section{INTRODUCTION}

$\mathbf{T}$ HE global temperature in February 2009 was the ninth warmest by $0.90{ }^{\circ} \mathrm{F}$ above the 20th century mean of $53.9^{\circ} \mathrm{F}[6]$. The increase in the global temperature is largely due to greenhouse gas emissions. These emissions could be reduced substantially by the conversion of gasoline combustion vehicles into electric vehicles (EVs), such as hybrid, plug-in hybrid, and battery EVs. For instance, the replacement of $77 \%$ of all transport miles with EVs will reduce carbon intensity by $94 \%$ over the 1990 numbers [20]. Also, due to soaring fuel prices, EVs are gaining popularity in the global market. To meet this global market demand, we need battery technologies that will make EVs cost- and efficiency-competitive with today's gasoline-powered vehicles.

Manuscript received October 23, 2010; accepted August 02, 2011. Date of publication September 06, 2011; date of current version April 11, 2012. Paper no. TII-10-10-0279

The authors are with the Real-Time Computing Laboratory, Department of Electrical Engineering and Computer Science, University of Michigan, Ann Arbor, MI 48109-2121 USA (e-mail: hahnsang.kim@gmail.com; kgshin@umich.edu).

Digital Object Identifier 10.1109/TII.2011.2166771
Cost-effective EVs require not only development of high energy-density battery cells, but also efficient management of large-scale battery packs, each consisting of a large number of battery cells for EVs, e.g., 6800 lithium-ion battery cells for Tesla Motors' EVs [12] and hundreds of cells for GM 2010 Chevy Volt [15]. In particular, a battery management system (BMS) that monitors and controls battery cells in a pack, must cope with heterogeneous battery-cell characteristics. That is, even if characteristics of all battery cells in a battery pack are initially identical, as they are charged and discharged repeatedly, each cell will exhibit different characteristics. A weak cell-that is charged and/or discharged faster than others-is likely to be overcharged and/or deep-discharged, i.e., the battery cell continues to be discharged even when its terminal voltage falls below a certain threshold called the cutoff voltage. This weak battery cell can eventually become faulty, and will, if not managed properly, cause the whole pack to be dysfunctional.

A BMS should be able to cope with weak/faulty cells in such a way that faulty cells are bypassed to keep the pack operational. Bypassing certain cells inside a pack, however, requires switches by which the connection arrangement of battery cells can be altered as described in [5], [7], [10], [18], and [19]. Switches are placed around each battery cell, regulating the battery supply power. Furthermore, the reconfigurable battery system we developed earlier [10] offers a way to alter battery connectivity and dynamically adjust supply power to meet application demands. All of these systems require careful system specification, cost-effective incorporation and control of system components, such as switches and battery cells.

One can conceive two types of battery management architecture: flat and modular. In the former, a single control module is responsible for monitoring and controlling all components. This architecture is easy to implement, but does not scale well; as the number of components to be monitored and controlled increases, the architectural complexity (e.g., wiring) and management latency grow rapidly. It is not energy-efficient, either. By contrast, in the modular architecture, an individual control module is only responsible for a subset of components independently or in cooperation. A prototypical design of modular system is presented in [17], consisting of four modules, each of which monitors a series-chain of battery cells. Such a distributed scheme makes monitoring more efficient, and energy-efficiency higher than the flat architecture. However, the cost of components increases, and the nature of the battery system requires a global module to orchestrate the others. A mere hierarchical 
system is neither effective nor efficient in monitoring and controlling battery dynamics. Therefore, we need smart management that makes the most of a reconfigurable framework. Such synergetic integration will maximize system performance and reliability at minimum cost.

There are two main challenges in developing a smart battery management architecture. First, there is a tradeoff between the minimum number of hardware components to use and maximum reconfigurability in a BMS. Key components therein are switches that allow a battery-cell array to be reconfigurable. The more switches around cells, the more reconfigurable the array becomes, but the costlier. Also, individual components affect directly system reliability. System reliability should be assessed based on the reliability of components and their connections. Since the cost is the major consideration in realizing a reconfigurable architecture, the components count should also be minimized. Second, to maximize both system reconfigurability and reliability, a reconfigurable architecture should be specified with respect to software/hardware components and their inter-relationship. An application (software) may require various battery (hardware) conditions from a BMS. Also, a BMS may request subsystem/local BMSs, if any, for the information on the status of individual battery cells in the case of modular management architecture. Upon receipt of this request, individual local BMSs periodically monitor their battery-cell arrays and reconfigure them, if necessary, in accordance with individual cell characteristics. This interaction between local BMSs also depends upon the underlying hardware system design. A well-designed, combined hardware-software architecture will provide high reliability, cost-effectiveness, and scalability.

We propose such an integration architecture for the management of large-scale battery packs, called the Dependable, Efficient, Scalable Architecture (DESA). Within DESA, BMSs are differentiated, according to their roles, as a single global BMS and multiple local BMSs. Each local BMS consists of a local controller, a set of switches (referred to as array-level switches), and a battery-cell array that includes a group of battery cells and a set of switches (referred to as cell-level switches). The global BMS, on the other hand, relies on a global controller. The global controller "speaks" to the local controllers so as to configure the array-level switches based on a switch-configuration algorithm, while individual local controllers configure celllevel switches within their battery-cell array. The relationship between the global and local BMSs is considered monarchybased. That is, the local BMSs govern their battery-cell array autonomously, while the global BMS controls array-level switches via local BMSs as needed. This hierarchical arrangement facilitates: (1) switch-configuration management, i.e., the policy for cell-level arrangement (by the local BMSs) is applied to array-level arrangement (by the global BMS); (2) achievement of system scalability, i.e., effectively coping with large-scale battery cells; and (3) improvement of power savings for the entire system by putting idle local BMSs into sleep mode.

\section{A. Key Contributions}

The main contributions of this paper are fourfold.

- DESA is designed to use the minimum number of switches while achieving reconfigurability - a battery-cell array (array-level) and battery cells therein (cell-level) can be rearranged online in parallel or in series while bypassing any battery cells or battery-cell arrays.

- DESA achieves scalability for a large-scale battery pack while providing a systematic switch configuration algorithm. This tightly coupled system provides synergetic performance typical of cyberphysical systems.

- Analytical results give a physical insight into the durability of switches, system reliability, system scalability, and service-cost savings. In particular, a fraction of the charge current load on individual switches varies with cell-level or array-level arrangement. This different fraction causes the switches to have different lifetimes. The proper choice of switches for different requirements greatly enhances the system reliability with respect to the battery lifetime, achieving service-cost savings.

- DESA is an energy-efficient architecture. It allows local BMSs including cell arrays to be selectively powered-off, depending on the level of load-demand, decreasing energy-dissipation across the entire BMS. Each power-off decision is therefore tightly-coupled with a real-time system configuration. For this configuration, the monitoring frequency, the cell arrangement, the system analysis are performed.

The rest of this paper is organized as follows. Section II describes DESA that consists of global and local BMSs, and other key components, including switches. We also present configuration commands and an algorithm to control switches. Section III presents the model of BMS power management using a Markov model and compare the performance of energy savings with DESA and a legacy system. Section IV presents the analysis of stress on each switch with respect to cell- and array-level connection arrangements. Section V presents a cost model for a battery-cell array for various types of battery and switch faults. Section VI evaluates the performance of DESA, and we conclude this paper in Section VII.

\section{DESA}

This section describes the architecture of DESA with details of its components.

\section{A. Overall Architecture}

As shown in Fig. 1, DESA consists of a global BMS and multiple local BMSs. The global BMS is formed by a global controller, a current meter, and controller area network (CAN)-based communication module [2]. A local BMS is formed by a controller, a set of array-level switches (i.e., P-, $\mathrm{S}$-, and B-switches), a voltage sensor, a battery-cell array, and a CAN module. Each local BMS monitors the battery condition, e.g., voltage, temperature, State-of-Charge (SoC) and State-of-Health $(\mathrm{SoH})$, and is connected to the global BMS via CAN.

The global and local BMSs have monarchy-based relationships. The global controller determines an array-level arrangement via a systematic switch configuration algorithm presented in Fig. 2. The local controllers execute command codes listed in Table I corresponding to the arrangement directed by the global controller. The local controllers can also determine their cell-level arrangement autonomously and which cell(s) to be bypassed. In the monarchy-based structure, each local controller 


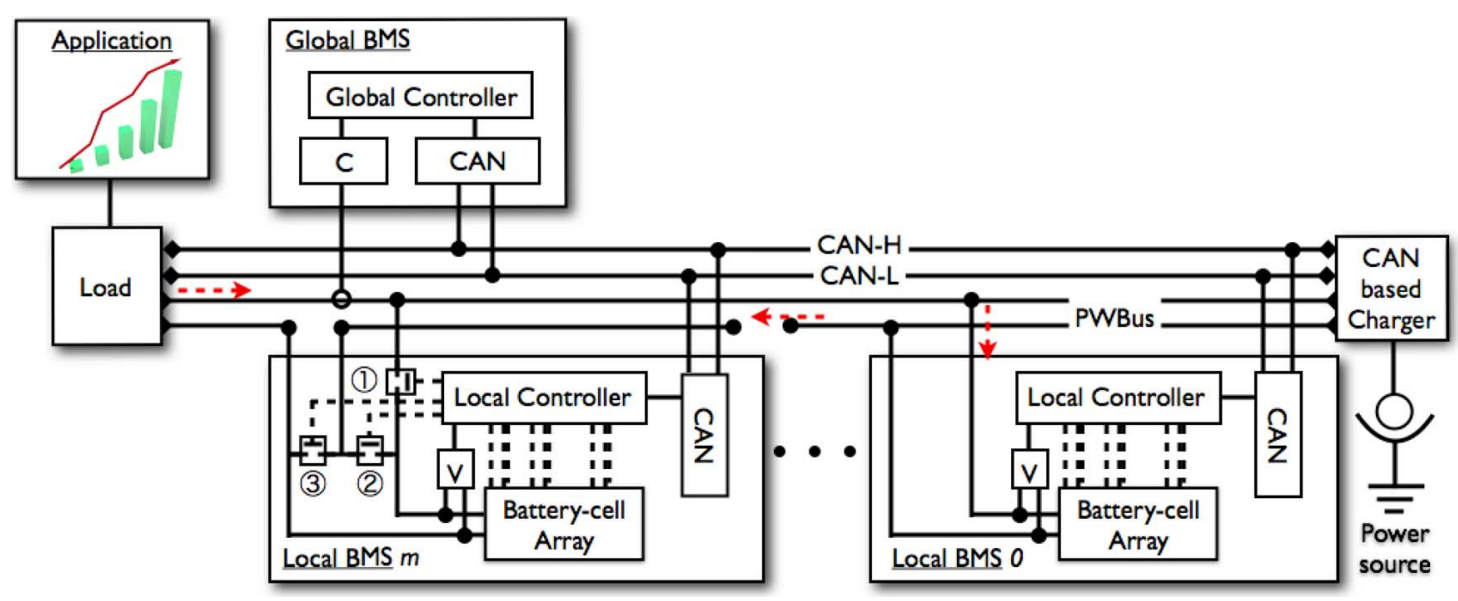

Fig. 1. The schematic diagram of DESA: switch (1) is called the P-switch, and switches (2) and (3) are called the S- and B-switches, respectively. The arrowed line indicates the discharge of the battery.

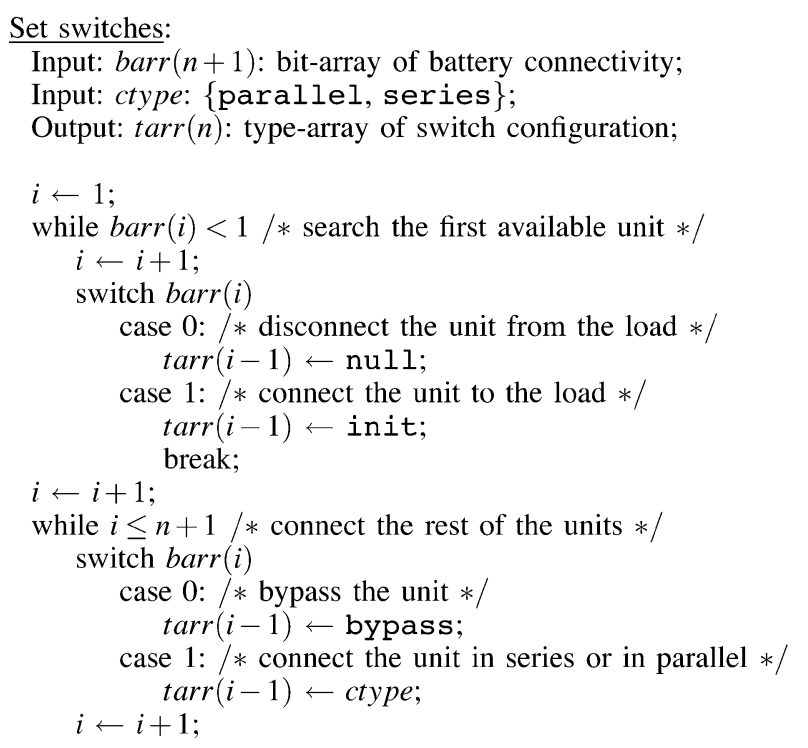

Fig. 2. Switch configuration algorithm.

TABEL I

COMMAND TYPE AND CODE

\begin{tabular}{|c||c|c|c|c|c|}
\hline Type & NULL & INIT & BYPASS & PARALLEL & SERIES \\
\hline Code & 000 & 100 & 001 & 101 & 010 \\
\hline
\end{tabular}

is responsible for monitoring its cells and responding to the global controller's interrogation. The monarchy-based structure is scalable to a large-scale battery pack by sharing with local controllers various tasks related to arranging, monitoring, and scheduling battery cells and arrays.

Alternatively, the relationship between the global and local controllers can be viewed as fully centralized, as the global controller determines both cell- and array-level arrangements. In the fully centralized structure, a local controller's role is minimum and hence unneeded; the global controller may directly monitor individual battery cells, bypass certain cells, and perform load-balancing for discharge, cell-balancing for charge, or voltage-balancing for both. In this sense, the fully centralized structure is agile in detecting and preventing an anomaly caused by some cells. This structure, however, can quickly be overwhelmed by a large number of battery cells to manage. As the number of cells increases, the time to monitor them will increase. Therefore, the monarchy-based structure may outperform the fully centralized beyond a certain number of battery cells.

In what follows, we detail the design of a battery-cell array and the characteristics of switches, a current meter, a thermistor, and a CAN.

1) Battery-Cell Array: A battery-cell array is designed by making the tradeoff between cost and reconfigurability, both of which increase monotonically with the number of switches used. By reconfigurability, we mean the capability of bypassing any battery cell, enabling effective voltage-balancing via selective discharge or charge of cells and extending the pack's operation-time even in the event of random cell failures. Besides, this capability will provide two types of reconfigurability. First, all cells in an array can be connected in parallel or in series. When they are connected in parallel (series), the array's capacity (terminal voltage) will be a cell's capacity (voltage) $\times$ the number of cells. Second, any individual cell can be charged separately, which is important for cell-balancing.

The number of switches required is determined based on connection arrangements. In the series arrangement, some weak (short-circuited) cell may have little effect on the array's current except that the overall terminal voltage may drop by no more than the weak cell's voltage. Some weak (open-circuited) cell, however, can block the current flow, making the series-chain of battery cells unusable. To bypass the weak cell, two switches (i.e., B- and S-switches), are placed as shown in the right side of Fig. 1. In the parallel arrangement, a single short-circuited cell makes the other cells unusable. In this case, a single switch (i.e., P-switch) can make the circuit opened. Consequently, three switches per cell are sufficient to bypass any cell in both parallel and series arrangements. The more customized the arrangement, the smaller the total number of switches required.

For switch failures, we adopt the well-known stuck-at fault model in which a faulty switch stays at either ON or OFF state permanently, irrespective of inputs to the switch. How to detect switch failures, however, is outside the scope of this paper. Instead, we assume that the local and the global controllers are 
capable of detecting these switch failures in a timely manner. Based on this fault model, the reliability of the entire battery pack will be analyzed in Section V.

2) Switch Characteristics: Reliable, robust switches are essential to withstand high voltages and currents. There are three switch modes: on, off, and transition. In the on mode, a switch is conductive and must have a low impedance, e.g., in the order of milli ohms. The lower the impedance, the lower the power dissipation, and hence, the lower the radiative heat. In the off mode, a switch is resistive and blocks high currents. High voltages can create a damaging electric arc, particularly at mechanical contacts. In the transition mode, a switch transits from on to off mode, or vice versa. A high voltage that can generate an electric arc, may destroy the switch circuit.

Considering the above switch characteristics, one must select proper switches. Two types of switch can meet our need: semiconductor and electromechanical relay switches. In the on mode, a switch's impedance is a key selection criterion. MOSFETs or IGBTs have relatively low impedance, so they may be appropriate for high current applications [1]. For instance, in MOSFETs, given the impedance of 5 milli ohms, a high current of 100 Amperes creates 50 Watts of power dissipation $\left(P=I^{2} \times R\right)$. By contrast, electromechanical relays are known to have a two orders-of-magnitude lower contact resistance than semiconductor switches, decreasing power dissipation to 0.5 Watt. In the off or transition mode, electromechanical relays can be weakened or even destroyed by arcs due to high voltages, while such an effect does not exist in semiconductor switches. To suppress such arcs, one may use additional hardware components that rely on magnetic fields.

It is, therefore, important to analyze current loads to be imposed on P-, S-, and B-switches, especially in cell- and arraylevel connection arrangements. Section IV will elaborate on this analysis.

3) Current Meter: A large range of time-varying currents requires fine-grained metering; a larger range leads to higher metering capability. A higher sampling rate yields a higher accuracy in measurements, but incurs higher cost; high output values at a high sample rate requires a high-performance processor/global controller. In general, a linear Hall sensor integrated with digital signal processing, such as TLE4998 [9], is widely used for highly-accurate measurements.

Current sensing is performed primarily by the global BMS and used for the estimation of battery SoC. In the array-level parallel arrangement, the global controller measures the current from the entire battery pack (Fig. 1), and then estimates the current of each local BMS by dividing the measured current by the number of arrays, assuming that all arrays are approximately identical with respect to their voltage and resistance values. When the discrepancy of these values exceeds a specified threshold, the estimate is no longer valid. In such a case, individual local BMSs should be able to measure them; the local controller measures the current from its battery cells in the same way as the global controller does at the array level. Obviously, there is a tradeoff between increase in accuracy and reduction in cost and workload. The measurement frequency is another design parameter for weighing the accuracy against the computation overhead. This issue will be treated in a separate forthcoming paper.
4) Thermistor: Each local BMS conducts thermal sensing. The main heat sources are battery cells, switches, and controllers. In particular, battery cells generate the most heat of all. Also, the battery capacity varies with ambient temperatures. Battery (e.g., lithium-ion) cells may not operate correctly as their temperature approaches $-58 \mathrm{~F}$, whereas they may explode if they approach $167 \mathrm{~F}$. Since the battery's operational temperature ranges very widely, we need to use a temperature sensor with accuracy on the order of $\pm 35 \mathrm{~F}$, which is available at an inexpensive cost. Digital temperature sensors, such as thermistors, thermocouples, and resistive temperature detectors [13], can meet our need. We also need a heat sink to which the dissipated heat is transferred. There are three basic modes of heat transfer-in a solid, in a fluid flow, and through a vacuum [14] — but the choice of heat sink is design-specific.

5) Can [2]: CAN is a message-based communication protocol by which all of the global controller, local controllers, and the charger broadcast and receive messages. When a certain node (e.g., a controller or a charger) finds messages of interest, it changes its mode dominant from recessive. The change in mode including sending messages is made via a pair of CAN High Speed (CAN-H) [3] and CAN Low Speed (CAN-L) [4] that form the physical layer of CAN. Since every node can put messages on, and must monitor, the CAN bus, collision may occur, in which case the message from the node with the highest ID is sent. A node that lost this arbitration waits until CAN becomes idle, and then resends the original message. On receipt of the corresponding acknowledgement, the message sending is completed.

The key features of using CAN include: 1) backward compatibility with existing nodes; 2) accommodation of as many CAN-based nodes as possible (e.g., a hundred nodes); 3) extensible capability of CAN such as 16-bit or 32-bit CAN; and 4) collision resolution with nondestructive bitwise arbitration. These salient features justify our choice of CAN for battery management.

\section{B. Command Codes for Controlling Switches}

Each command code is 3 digits long. The first digit indicates the P-switch's state, and the second and the last digits indicate states of the S- and B-switches, respectively. The value of 0 (1) means that the corresponding switches are turned off (on).

These codes are designed to control P-, S-, and B-switches, and applied for both the array- and cell-level configurations. At the array-level configuration, the global controller issues an appropriate command in Table I to individual local controllers. A sequence of commands are issued systematically based on the systematic switch configuration algorithm shown in Fig. 2. The local controllers then execute the dispatched command to control the array-level switches. At the cell-level configuration, on the other hand, the local controller executes a sequence of commands on individual cells independently.

Each command has its own purpose. First, the NULL code is applied to keep a specific battery cell (array) open, disconnecting all the battery cells (arrays) behind the cell. For instance, setting Cell 2 to the NULL code means that Cells 0 and 1 are not in use, irrespective of the configuration of their switches. This bypass can be an option for the case that any switches around Cells 0 and 1 are dysfunctional. Second, the INIT code indicates 
the beginning of the battery-cell array (a chain of arrays). Thus, the INIT code is applied to the battery cell (array) next to the one to which the NULL code has been applied. Third, the BYPASS code is applied to bypass any battery cell (array) except for the first, i.e., Cell 0 (local BMS 0). Since the first cell (local BMS 0 ) does not have its own switches, the INIT code is applied to the next cell, bypassing the first. Next, the PARALLEL code is applied to make a parallel arrangement. Likewise, the SERIES code is applied to make a series arrangement.

\section{Data Aggregation}

The global BMS periodically aggregates the information on battery conditions (including voltage, temperature, and current) that individual local BMSs monitor cells within their batterycell array. The local controller measures a terminal voltage between the two terminals of the battery-cell array. On the other hand, to measure the voltage of individual cells, e.g., Cell $i$, the local controller applies the INIT code to Cell $i$, and the BYPASS code to Cell $i+1$ to $n$. In case of Cell 0 , it applies the BYPASS code to all cells. The global controller then fetches the voltage measurement from the local controller during the aggregation period. The local controller also measures the temperature of its battery-cell array. When the temperature exceeds a certain threshold, the local controller disconnects the cell from the load by issuing the NULL code to Cell $n$. Unlike the voltage measurement, the local controller reports this anamoly to the global controller whenever it occurs. For the current measurement, it is delegated to the global BMS, since it is costly.

\section{Array-and Cell-Level Arrangements}

The global BMS is responsible for the array-level arrangement, while the local BMS is for the cell-level arrangement. According to the switch configuration algorithm in Fig. 2, the parallel arrangement is changed to the series arrangement or vice versa. First, the global (local) controller takes as an input a bitarray of connectivity, barr, where 1 and 0 of the $i$ th bit indicates the connection and bypassing of the $i$ th array (cell), respectively. Then, it searches for the first available array (cell) and applies the INIT command to the array-level (cell-level) switches. Thereafter, when arrays (cells) are to be connected in parallel or in series, the PARALLEL or the SERIES code is applied to individual arrays (cells), respectively. Thus, the arrays (cells) are connected as specified.

During the course of battery activities (i.e., charge, discharge, and rest), some arrays (cells) may be bypassed. In this case, the global (local) controller determines which arrays (cells) to be bypassed by setting barr, and then runs the algorithm with barr. The local BMSs that have the arrays bypassed may be put into sleep mode where the monitoring halts to save power. The local BMSs in the sleep mode go back to the operation mode upon the global controller's request. The rest period depends on a discharge rate; the lower the discharge rate, the longer the rest period. It is determined based on a battery-activity scheduling mechanism in [11].

The local controllers running the switch configuration algorithm can effectively perform voltage-balancing based on the scheduling mechanism. Also, the local controller can autonomously decide to make its battery-cell array open-circuited by applying the NULL command to the array-level switches. This is an exceptional case: the array is overheated, overcharged, or deep-discharged.

\section{E. Interaction With On-Board Applications}

The application requests, from the global BMS, the battery's remaining operation-time, the time for the battery to fully be charged, and the battery's lifetime - in particular, accurate prediction of the lifetime is of great importance to the battery's lifetime warranty. First, for the battery's operation-time, the global BMS feeds the aggregated voltage and current into a reference model [11]. This reference model includes functions of time-dependent charge and discharge rates. Applying the reference model results in the remaining operation-time. Second, as in the calculation of the battery's operation-time, the time for the battery to be fully charged is also obtained from the reference model with the current charge rate. Third, the battery's lifetime is assessed based on the internal impedance of the battery cells, since a high impedance dissipates high power, generating heat. This will ultimately lower the supply voltage and its effect will propagate to other battery cells. Such irreversible degradation will shorten the battery's lifetime. To assess the lifetime, we measure the battery's terminal voltage, computing the battery's internal impedance, such that $V=(R) /(R+r) V_{0}$, where $V_{0}$ is the reference voltage, and $R$ and $r$ are the load and the internal impedance, respectively. Note that various methods are available for this purpose.

\section{ENERGY-SAVING BATTERY MANAGEMENT}

DESA can adjust the power activity of the battery to the load demand by reconfiguring battery cell/array connections. This adjustment is to realize energy-savings achievable for individual battery-cell arrays or local BMSs. For instance, when the load demand is low enough to disconnect a battery-cell array for a certain period of time, the corresponding local BMS will be switched into a low-power mode, where minimum components such as the B-switch and the local controller are kept active (see Fig. 1). On the other hand, the adjustment in the power activity is highly dependent on the load demand that varies with road conditions and the user's driving style (which is somewhat predictable).

Here, we present DESA's energy-saving battery management by modeling power activity with a Markov chain. This modeling provides an analytical insight into the importance of energy-savings over legacy BMSs as well as a way to evaluate the DESA's energy-efficiency.

\section{A. Modeling}

We develop a Markov model, as shown in Fig. 3, to capture battery management actions. Each state in the model consists of a pair of values, $(i, j)$, where $i$ and $j$ refer to the numbers of active cell arrays and of available cell arrays, respectively. The state $(0,0)$ represents the initial stage of battery management. This state corresponds to the case where the battery is fully discharged. The state $(0, j)$ corresponds to cases where the battery is charged, assuming that the battery has $n$ levels of charge. So, when $j=n$, the battery is fully charged. The state $(i, j)$, where $i<j$, captures the energy-savings of battery management, in 


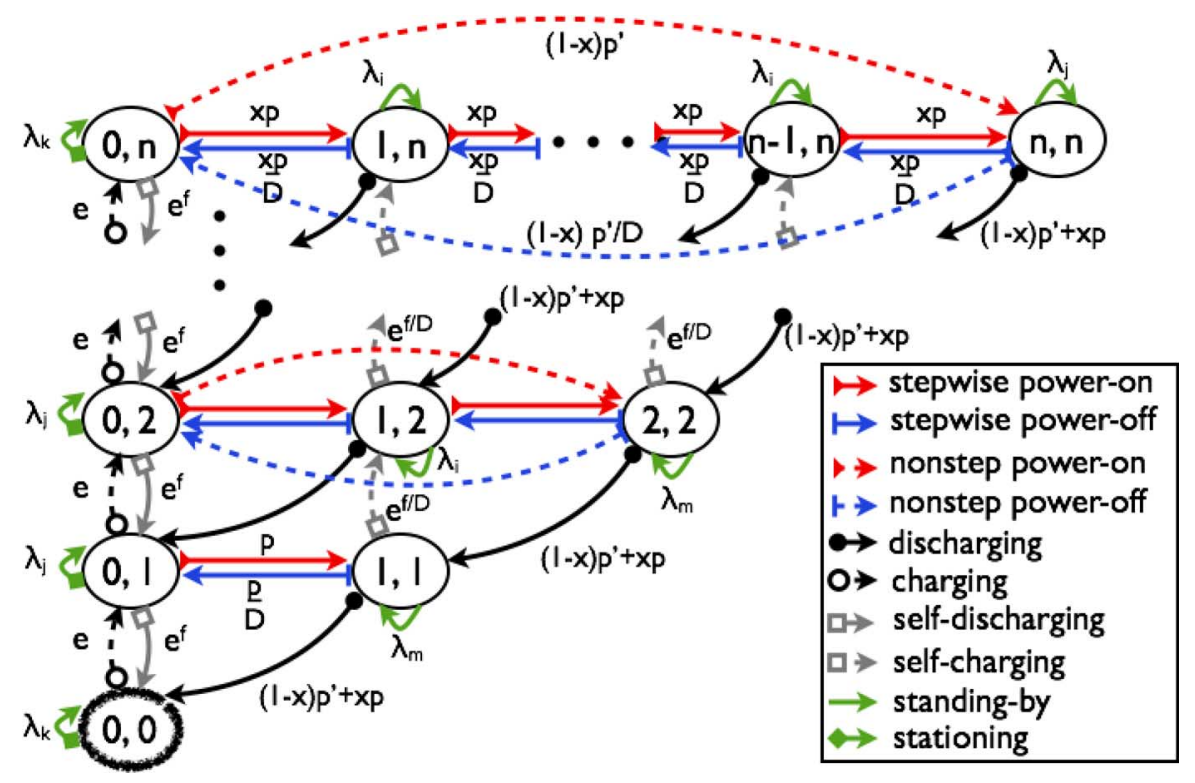

Fig. 3. A Markov model for system power management.

proportion to $(j-i)$. Cases where $i=j$ correspond to the state that all cell arrays are being used.

The state transitions in the model are illustrated in Fig. 3 with different line styles indicating different events (power plug-in, power-on, power-off, pedal-breaking, pedal-acceleration, etc.) that cause state transitions. The system parameters considered in the state transitions are:

- $1 / e$ : battery charging rate;

- $p$ and $p^{\prime}$ : interval of load-demand arrivals at the battery;

- $D$ : timeout timer value for energy-savings;

- $f$ : battery impedance factor.

In addition, we use the following parameters related to self-transitions:

- $\lambda_{k}$ and $\lambda_{j}$ : battery-charging delay;

- $\lambda_{i}$ and $\lambda_{m}$ : load-demand arrival delay.

We model the battery charging rate between charging states as an exponentially-distributed random variable (with mean $e$ ), the load-demand arrival interval as a Poisson process with rate $1 / p$ for a DESA-embedded system $\left(1 / p^{\prime}\right.$ for a legacy BMS), and timeout timer values and impedance factors as exponentially distributed random variables with means $D$ and $f$, respectively. Furthermore, we approximate the battery-charging and load-demand arrival delays as exponentially-distributed random variables with means $1 / \lambda_{k}$ and $1 / \lambda_{j}$, and $1 / \lambda_{i}$ and $1 / \lambda_{m}$, respectively.

As briefly mentioned before, the Markov chain shown in Fig. 3 encompasses the DESA and non-DESA (legacy) system. The former is modeled with $x=1$ and the latter is with $x=0$. We next describe the state transitions shown in the figure.

In a DESA-equipped system (i.e., $x=1$ ), power deliverable from the battery is gradually increased, according to the load demand. For this, cell arrays and their local BMSs are turned on stepwise (from $(i, j)$ to $(i+1, j)$ ). Conversely, the deliverable power is decreased as the load demand dwindles. Since the load demand is estimated, individual cell arrays can actually be overdischarged during a short period of time. To mitigate such an unnecessary stress on batteries, DESA uses a timer which is set by a decrease in the load demand. When the timer expires, DESA powers off an active cell array. DESA allows the battery to be simultaneously charged and discharged as long as the power source for such concurrent charging is provided, which is represented by the transition from $(i, j)$ to $(i, j+1)$. In a legacy system (i.e., $x=0$ ), on receipt of a load demand, all arrays within the battery are turned on simultaneously (from $(0, j)$ to $(n, n))$. Similarly, when there is no load demand, all arrays are turned off. The battery in both systems is either self-discharged, or discharged by the load. Self-discharging occurs as the battery ages, most likely resulting in high impedance. These two actions are represented by transitions from $(0, j)$ to $(0, j-1)$ and from $(i, j)$ to $(i-1, j-1)$, respectively.

\section{B. Model Solution}

Using this model, we can study the performance of DESA. Specifically, we are interested in system energy-efficiency rate, $\delta$, defined as the power consumption of DESA divided by that of the legacy BMS. To obtain $\delta$, we need to know the fraction of time that the system stays in states $(i, j)$, where $i>0$ and $j>0$, and also assume that the power consumption in each of these states is proportional to $i$. This is equivalent to evaluating the sum of the stationary probabilities of the model states. Let $\pi_{i, j}$ be the stationary probability of being in state $(i, j)$. We have the following expression for $\delta$ :

$$
\delta=1-\frac{\sum_{0<i \leq j \leq n} i \cdot \pi_{i, j}}{\sum_{0<j \leq n} j \cdot \pi_{j, j}} .
$$

\section{Model Evaluation}

We now compare and contrast the performance of the two systems. In order to use representative parameter values, we first calculate local optimal values of the system parameters used in the model as: $e=0.1, f=6, p=0.3, p^{\prime}=p$, and $D=2$. 


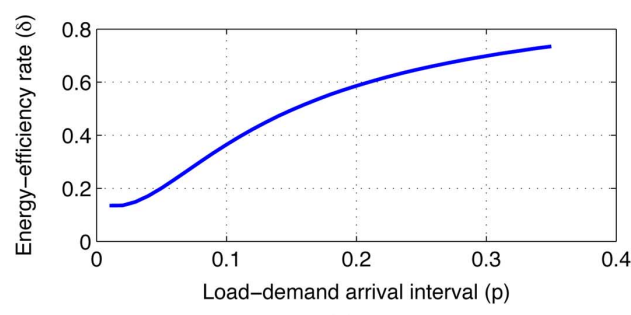

(a)

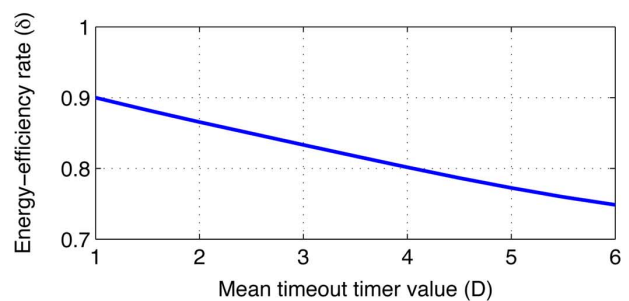

(b)

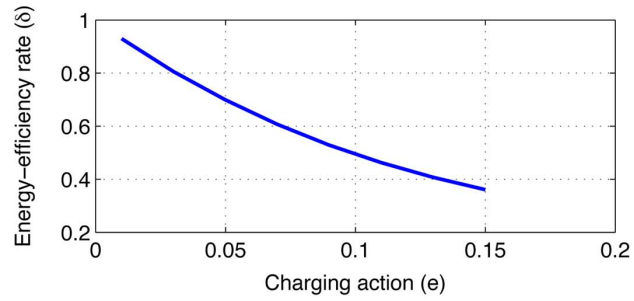

(c)

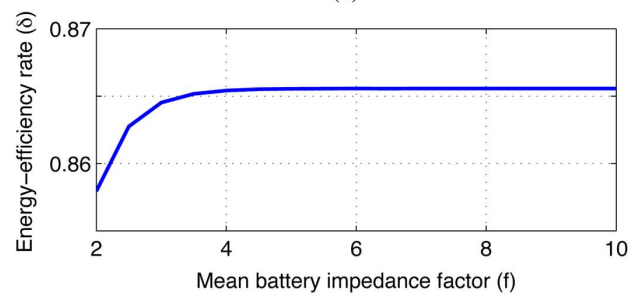

(d)

Fig. 4. System energy-efficiency rates with respect to representative parameters. (a) Increase in efficiency with respect to load demand. (b) Linear decrease in efficiency with respect to energy-savings. (c) Linear decrease in efficiency with respect to charging. (d) Exponential increase in efficiency with respect to impedance.

Unless otherwise noted, these values are used throughout our evaluation.

Fig. 4 provides a number of insights into the DESA-equipped system. First, when the expected interval of load-demand arrivals at the battery increases, the system energy-efficiency rate, $\delta$, also increases. When $p<0.1, \delta$ exponentially increases, and then continuously goes up, e.g., with a gradient of 3.3 between 0.1 and $0.2(=p)$ shown in Fig. 4(a). That is, the efficiency gain by the DESA increases proportionally to the degree of its use. Second, a conservative approach to energy-savings - the expected timeout timer value gets larger-makes a minor impact on $\delta$, e.g., with a gradient of less than 0.05 between 2 and $4(=D)$ shown in Fig. 4(b). However, since the future load demand is only an estimation, aggressive energy-savings can stress currently active cell arrays. Next, frequent charging does not achieve high efficiency. For instance, in Fig. 4(c), when the charging rate is high (e.g., $e=0.05$ ), $\delta$ gets as large as 0.7 , whereas the value of $\delta$ drops by $28 \%$ when $e=0.1$. Finally,
TABLE II

CURRENT ON ARRAY-LEVEL SWITCHES IN THE $(k+1)$ TH LOCAL BMS

\begin{tabular}{|c|c|c|c|}
\hline Arrangement & P-switch & S-switch & B-switch \\
\hline \hline Parallel & $(1-p) \frac{x}{r} \mathrm{C}$ & $0 \mathrm{C}$ & $(1-p) \frac{i x}{r} \mathrm{C}$ \\
\hline Series & $p^{k}(1-p) x \mathrm{C}$ & $(1-p) x \mathrm{C}$ & $p x \mathrm{C}$ \\
\hline
\end{tabular}

the expected battery impedance barely affects the energy-efficiency. For instance, in Fig. 4(d), the efficiency gain from 2 to 4 $(=f)$ is less than $1 \%$, and when $f>4, \delta$ rarely changes. This represents a real-life battery characteristic.

\section{POWER DisSIPATION ON SWITCHES}

As mentioned earlier, given constant resistance inside a switch, power dissipation on switches exponentially increases as the current passing through it increases. Thus, it is essential to assess the current through switches, which is analyzed in this section for the array- and cell-level arrangements.

\section{A. Current Load on Switches at Array Level}

The current loads on array-level P-, S-, and B-switches are differentiated in parallel and series arrangements. For simplicity, we assume that all arrays are identical. The parameters used in our analysis are:

- $x \mathrm{C}$ : coulombs (per second) required by the load;

- $p$ : probability of a local BMS being bypassed;

- $r \leq N$ : number of local BMSs connected to the load for charge or discharge among $N$ arrays in total;

- $k \leq r$ : number of local BMSs consecutively connected among $r$ BMSs;

- $i$ : number of local BMSs among $k$ local LMSs that precede the $(k+1)$ th local BMS.

In the parallel arrangement, the array-level P-switch in each local BMS (Fig. 1) is switched on over the course of battery charge or discharge. Since the current load is equally shared with $r$ local BMSs, the P-switch is loaded with $(x / r) \mathrm{C}$ with the probability of $(1-p)$. The array-level $\mathrm{S}$-switch is never switched on, while the array-level B-switch serves as the conductors. Thus, in a customized battery-cell array, the S- and B-switch can be removed and replaced with a wire. Otherwise, the current load on the B-switch proportionally increases as $i$ increases, resulting in $(i x) /(r) \mathrm{C}$ with the probability of $(1-p)$.

In the series arrangement, the array-level S-switch in each local BMS is always switched on unless the local BMS thereof is bypassed. The currents on these $\mathrm{S}$-switches are the same, resulting in $x \mathrm{C}$ with the probability of $(1-p)$. The array-level $\mathrm{P}$-switch in a local BMS, on the other hand, is switched on only if all preceding local BMSs are bypassed. Thus, the current on the P-switch in the $(k+1)$ th local BMS is equal to $p^{k}(1-$ $p) x \mathrm{C}$. The array-level B-switch in a local BMS is switched on only if the local BMS is to be bypassed with the probability of $p$, resulting in $p x \mathrm{C}$. Table II shows the current load on each array-level switch in the $(k+1)$ th local BMS.

\section{B. Current Load on Cell-Level Switches}

Cell-level switches are current-loaded as array-level switches. At the cell level, however, the current fed into a battery-cell array varies with the array-level arrangement. In the array-level parallel arrangement, the array-level P-switch is current-loaded directly, i.e., $\mathrm{C}^{*}=(1-p)(x / r) \mathrm{C}$, whereas 
TABLE III

CURRENT ON CELL-LEVEL SWITCHES IN THE $(l+1)$ TH LOCAL BMS: $\mathrm{C}^{*}$ AND $^{+} \mathrm{C}^{+}$ARE INPUT CURRENTS DETERMINED BY THE ARRAY-LEVEL ARRANGEMENT, I.E., ARRAY-LEVEL P-SWITCHES

\begin{tabular}{|c|c|c|c|}
\hline Arrangement & P-switch & S-switch & B-switch \\
\hline \hline Parallel (Parallel) & $\frac{(1-q)}{s} \mathrm{C}^{*}$ & $0 \mathrm{C}$ & $(1-q) \frac{j}{s} \mathrm{C}^{*}$ \\
\hline Parallel (Series) & $\frac{(1-q)}{s} \mathrm{C}^{+}$ & $0 \mathrm{C}$ & $(1-q) \frac{j}{s} \mathrm{C}^{+}$ \\
\hline Series (Parallel) & $q(1-q) \mathrm{C}^{*}$ & $(1-q) \mathrm{C}^{*}$ & $q \mathrm{C}^{*}$ \\
\hline Series (Series) & $q^{l}(1-q) \mathrm{C}^{+}$ & $(1-q) \mathrm{C}^{+}$ & $q \mathrm{C}^{+}$ \\
\hline
\end{tabular}

the array-level S-switch is current-loaded in the array-level series arrangement, i.e., $\mathrm{C}^{*}=(1-p)(x / r) \mathrm{C}$ in the array-level series arrangement. Given $\mathrm{C}^{*}$ and $\mathrm{C}_{+}$, the current load on each cell-level switch is determined. The parameters to be used are:

- $q$ : probability of a cell in the battery-cell array being bypassed;

- $s \leq n$ : number of cells connected in a battery-cell array for charge or discharge among a total of $n$ cells;

- $l \leq s$ : number of cells connected consecutively in a battery-cell array;

- $j$ : number of cells among $l$ cells which precede the $(l+1)$ th cell.

In the parallel arrangement, each cell-level P-switch within a battery-cell array is switched on during the course of battery charge or discharge. The current load is equally shared with $s$ battery cells, resulting in $(1 / s) \mathrm{C}^{*}\left(\mathrm{C}^{+}\right)$in the array-level parallel (series) arrangement with the probability of $(1-q)$. Cell-level S-switches, on the other hand, are never switched on, while cell-level B-switches serve as the conductors. Like the corresponding array-level switches, they are removed or replaced with a wire. Otherwise, the current load on the cell-level $\mathrm{B}$-switch proportionally increases as $j$ increases, resulting in $(j / s) \mathrm{C}^{*}\left(\mathrm{C}^{+}\right)$in the array-level parallel (series) arrangement with the probability of $(1-q)$.

In the series arrangement, the cell-level S-switch in each cell is always switched on unless the corresponding cell is bypassed. The current loads on these $\mathrm{S}$-switches are the same, resulting in $\mathrm{C}^{*} / \mathrm{C}^{+}$with the probability of $(1-q)$. The current loads on the array-level P- and B-switches are also obtained in the same way as in the array-level series arrangement. Table III shows the current load on each cell-level switch in the $(l+1)$ th cell.

\section{COST MOdel FOR A BATtery PACK}

To assess the cost-effectiveness of a battery pack, we define its total cost $C_{T}$ as the sum of manufacturing and service costs [16] as

$$
C_{T}=C_{M}+C_{S}
$$

where $C_{M}$ and $C_{S}$ are the manufacturing and the service costs, respectively. $C_{M}$ is closely related to the imperfect testing process, whereas $C_{S}$ depends on the reliability of a battery pack within its warranty period. To assess reliability, we use a simple battery fault model: a battery cell fails when it becomes open-circuited (denoted as $F_{B}=0$ ) or short-circuited (denoted as $F_{B}=1$ ). Similarly, in the stuck-at fault model, a switch fails when it is stuck-at ON (denoted as $F_{\mathrm{SW}}=1$ ) or OFF state (denoted as $F_{\mathrm{SW}}=0$ ), regardless of input.

In what follows, we elaborate on the cost model.

\section{A. Manufacturing Cost Model}

Before shipping products to customers, it is important for the manufacturer to test them, since their failure in the field incurrs significant expenses and influences the customer's satisfaction or the manufacturer's reputation. Suppose that $N$ battery-cell arrays form a battery pack and the observed yield per pack is $y_{a}$, then the manufacturing cost per pack can be modeled as

$$
C_{M}=\frac{N \cdot C_{A}}{y_{a}}
$$

The yield is the probability that a battery pack passes the test. This probability depends upon the fault coverage $F$; when $F=$ 0 , no fault occurs. Using a negative binomial yield model [8], [16], this probability is expressed as

$$
y_{a}\left(F, \lambda_{a}, \alpha\right)=\left(1+\frac{F \cdot \lambda_{a}}{\alpha}\right)^{-\alpha}
$$

where $\lambda_{a}$ is the average number of defects per array and $\alpha$ represents the degree to which defects are clustered. When $\lambda_{a}=0$, the battery-cell array is defect-free. When $\alpha \rightarrow 0$, defects are strongly clustered, while $\alpha \rightarrow \infty$ corresponds to weak clustering.

\section{B. Service Cost Model}

A battery pack may fail before the warranty expires. Even when some battery-cell arrays in the pack fail, the pack can "operate" ${ }^{1}$ with spare arrays in both DESA and a conventional BMS without any reconfigurable switch. A battery pack consists of $N$ battery-cell arrays, each of which is composed of $n$ battery cells. We assume that the battery pack can operate as long as at least $M$-out-of- $N$ arrays function, and each array operates as long as at least $m$-out-of- $n$ battery cells function. This assumption applies to both DESA and conventional BMSs. The failure of a battery pack will incur a service cost for its repair or replacement, which is typically overpriced. The service cost is then directly related to the pack's reliability and can be modeled as

$$
C_{S}=\left(1-R_{P}(t) y_{a}\right) C_{F}
$$

where $C_{F}$ is the service cost per pack, and $R_{P}(t)$ is the reliability (probability) that at least $M$ battery arrays in the pack are still operational at time $t . R_{P}(t)$ depends on the reliability of individual arrays. Let $R_{A}(t)$ be the reliability that a battery-cell array operates at time $t$, subject to individual components, i.e., battery cells and switches. Also, let $X_{B}\left(X_{\mathrm{SW}}\right)$ be an exponentially distributed random variable for a battery cell (switch) with rate $\lambda_{B}\left(\lambda_{\mathrm{SW}}^{*}\right)$. Then, $P\left\{X_{B}>t\right\}=e^{-\lambda_{B} t}$ and $P\left\{X_{\mathrm{SW}}>t\right\}=e^{-\lambda_{\mathrm{SW}}^{*} t}$. For simplicity of analysis, all battery cells (switches) are assumed identical.

Since $R_{P}(t)$ varies with the underlying fault model and battery arrangement, it is calculated with respect to each failure mode such that $\left(F_{B}, F_{\mathrm{SW}}\right)=\{(x, y) \mid x, y \in\{0,1\}\}$ and arraylevel parallel and series arrangements, resulting in 8 combinations. Each combination is divided into two parts, i.e., cell-level parallel and series arrangements. To indicate these configurations, we use notation CN.xy where $N$ denotes a configuration, and $x$ and $y$ the array-level and the cell-level arrangements,

\footnotetext{
${ }^{1}$ Providing the required voltage, current, or power.
} 
TABLE IV

COMPARISON OF RELIABILITY OF DESA AND THE CONVENTIONAL BMS

\begin{tabular}{|c|c|c|c|}
\hline Conf. $\left(F_{B}, F_{S W}\right)$ & Reliability of DESA & $\lambda_{S W}^{*}$ & Reliability of the conventional BMS \\
\hline C1.p $(1,1)$ & $\begin{aligned} R_{P}(t)= & 1-\left\{\sum_{i=1}^{n}\left(\begin{array}{l}n \\
i\end{array}\right)\left(P\left\{X_{S W} \leq t\right\}\left(1-R_{A}(t)\right)\right)^{i}\left(1-P\left\{X_{S W} \leq t\right\}\left(1-R_{A}(t)\right)\right)^{n-i}\right. \\
& \left.+\sum_{i=m+1}^{n}\left(\begin{array}{c}n \\
i\end{array}\right)\left(P\left\{X_{S W}>t\right\}\left(1-R_{A}(t)\right)\right)^{i}\left(1-P\left\{X_{S W}>t\right\}\left(1-R_{A}(t)\right)\right)^{n-i}\right\}\end{aligned}$ & $\lambda_{S W} \frac{1}{\tau} \frac{(1-p)}{M} x$ & $R_{P}(t)=\Pi_{i=1}^{N} R_{A}(t)$ \\
\hline C1.pp $(1,1)$ & $\begin{aligned} R_{A}(t)= & 1-\left\{\sum_{i=1}^{n}\left(\begin{array}{c}n \\
i\end{array}\right)\left(P\left\{X_{S W} \leq t\right\} P\left\{X_{B} \leq t\right\}\right)^{i}\left(1-P\left\{X_{S W} \leq t\right\} P\left\{X_{B} \leq t\right\}\right)^{n-i}\right. \\
& \left.+\sum_{i=m+1}^{n}\left(\begin{array}{c}n \\
i\end{array}\right)\left(P\left\{X_{S W}>t\right\} P\left\{X_{B} \leq t\right\}\right)^{i}\left(1-P\left\{X_{S W}>t\right\} P\left\{X_{B} \leq t\right\}\right)^{n-i}\right\}\end{aligned}$ & $\lambda_{S W} \frac{1}{\tau} \frac{(1-q)(1-p)}{m M} x$ & $R_{A}(t)=\Pi_{i=1}^{n} P\left\{X_{B}>t\right\}$ \\
\hline C1.ps $(1,1)$ & $R_{A}(t)=\sum_{i=m}^{n}\left(\begin{array}{c}n \\
i\end{array}\right) P\left\{\min \left(X_{B}, X_{S_{C}}\right)>t\right\}^{i}\left(1-P\left\{\min \left(X_{B}, X_{S_{C}}\right)>t\right\}\right)^{n-i}$ & $\lambda_{S W} \frac{1}{\tau} q \frac{(1-p)}{M} x$ & $R_{A}(t)=\sum_{i=m}^{n}\left(\begin{array}{c}n \\
i\end{array}\right) P\left\{X_{B}>t\right\}^{i}\left(1-P\left\{X_{B}>t\right\}\right)^{n-i}$ \\
\hline C2.s $(1,1)$ & $R_{P}(t)=\sum_{i=M}^{N}\left(\begin{array}{c}N \\
i\end{array}\right)\left(R_{A}(t) P\left\{X_{S_{A}}>t\right\}\right)^{i}\left(1-R_{A}(t) P\left\{X_{S_{A}}>t\right\}\right)^{N-i}$ & $\lambda_{S W} \frac{1}{\tau} p x$ & $R_{P}(t)=\sum_{i=M}^{N}\left(\begin{array}{c}N \\
i\end{array}\right) R_{A}(t)^{i}\left(1-R_{A}(t)\right)^{N-i}$ \\
\hline C2.sp $(1,1)$ & C1.pp & $\lambda_{S W} \frac{1}{\tau} \frac{(1-q)(1-p)}{m} x$ & C1.ps \\
\hline C2.ss $(1,1)$ & C1.ps & $\lambda_{S W} \frac{1}{\tau} q(1-p) x$ & C1.ps \\
\hline C3.p $(1,0)$ & $\mathrm{C} 2 . \mathrm{s}$ & $\lambda_{S W} \frac{1}{\tau} \frac{(1-p)}{M} x$ & C1.p \\
\hline C3.pp $(1,0)$ & C1.ps & $\lambda_{S W} \frac{1}{\tau} \frac{(1-q)(1-p)}{q M} x$ & C1.pp \\
\hline C3.ps $(1,0)$ & C1.ps & $\lambda_{S W} \frac{1}{\tau} \frac{(1-q)(1-p)}{M} x$ & C1.ps \\
\hline C4.s $(1,0)$ & $\mathrm{C} 2 . \mathrm{s}$ & $\lambda_{S W} \frac{1}{\tau}(1-p) x$ & $\mathrm{C} 2 . \mathrm{s}$ \\
\hline C4.sp $(1,0)$ & C1.ps & $\lambda_{S W} \frac{1}{\tau} \frac{(1-q)(1-p)}{m} x$ & C1.pp \\
\hline C4.ss $(1,0)$ & C1.ps & $\lambda_{S W} \frac{1}{\tau}(1-q)(1-p) x$ & C1.ps \\
\hline C5.p $(0,1)$ & The same as conventional BMS's & - & $\mathrm{C} 2 . \mathrm{s}$ \\
\hline C5.pp $(0,1)$ & The same as conventional BMS's & - & C1.ps \\
\hline C5.ps $(0,1)$ & C1.ps & $\lambda_{S W} \frac{1}{\tau} \frac{q(1-p)}{M} x$ & C1.pp \\
\hline C6.s $(0,1)$ & $\mathrm{C} 2 . \mathrm{s}$ & $\lambda_{S W} \frac{1}{\tau} p x$ & C1.p \\
\hline C6.sp $(0,1)$ & C5.pp & - & C1.ps \\
\hline C6.ss $(0,1)$ & C1.ps & $\lambda_{S W} \frac{1}{\tau} q(1-p) x$ & C1.pp \\
\hline C7.p $(0,0)$ & $\mathrm{C} 2 . \mathrm{s}$ & $\lambda_{S W} \frac{1}{\tau} \frac{(1-p)}{M} x$ & $\mathrm{C} 2 . \mathrm{s}$ \\
\hline C7.pp $(0,0)$ & C1.ps & $\lambda_{S W} \frac{1}{\tau} \frac{(1-q)(1-p)}{m M} x$ & C1.ps \\
\hline C7.ps $(0,0)$ & C1.ps & $\lambda_{S W} \frac{1}{2} \frac{(1-q)(1-p)}{M} x$ & C1.pp \\
\hline C8.s $(0,0)$ & $\mathrm{C} 2 . \mathrm{s}$ & $\lambda_{S W} \frac{1}{\tau}(1-p) x$ & C1.p \\
\hline C8.sp $(0,0)$ & C1.ps & $\lambda_{S W} \frac{1}{\tau} \frac{(1-q)(1-p)}{m} x$ & C1.ps \\
\hline C8.ss $(0,0)$ & C1.ps & $\lambda_{S W} \frac{1}{\tau}(1-q)(1-p) x$ & C1.pp \\
\hline
\end{tabular}

respectively. Table IV lists all the configurations with fault instances.

The reliability of a battery pack with DESA is compared with that the conventional BMS. In the parallel arrangement, a short-circuited battery cell (i.e., $F_{B}=1$ ) creates a domino effect, causing the failure of the entire battery pack to be dysfunctional. This effect is fatal to the conventional BMS. Thus, the system reliability in the conventional BMS is subject to that of individual battery cells, resulting in C1.p for the array-level and C1.pp for the cell-level in Table IV. By contrast, DESA effectively deters the total failure via switch setting, in which $\mathrm{P}$-switches play a critical role. In case switches get stuck-at ON state (i.e., $F_{\mathrm{SW}}=1$ ), even if a battery cell (array) fails, the entire array (pack) still operates as long as the P-switch functions and at least $m$-out-of- $n$ cells ( $M$-out-of- $N$ arrays) function; only when both the battery cell (array) and the corresponding P-switch fail, the array (pack) fails, resulting in the reliability of C1.pp for the cell-level (that of C2.p for the array-level). In case switches get stuck-at OFF state (i.e., $F_{\text {SW }}=0$ ), these switches can lower the reliability of battery cells (arrays). Thus, the minimum of a battery cell's (array's) lifetime and the corresponding P-switch's lifetime determines the reliability of the array (pack), resulting in that of C3.p for the cell-level (that of C3.pp for the array-level). On the other hand, an open-circuited battery cell (i.e., $F_{B}=0$ ) seldom affects the operation of the entire array except its output voltage drops no less than that of the cell itself. Thus, the array (pack) operates as long as at least $m$-out-of- $n$ cells ( $M$-out-of- $N$ arrays) operate, resulting in the reliability of C5.p for the cell-level (C5.pp for the array-level).
In the series arrangement, on the other hand, a short-circuited battery cell has the same effect as an open-circuited cell in the parallel arrangement on the reliability of the array (see C2.s and C2.ss). An open-circuited battery cell is critical since it can cause the entire array (pack) to be dysfunctional in the conventional BMS. This effect is the same as a short-circuited cell in the parallel arrangement. DESA bypasses the open-circuited cell by turning on the corresponding B-switch and off the S-switch. In case switches get stuck-at ON state, the B-switch is critical to reliability. In such a case, the cell (array) becomes open-circuited and the entire array's (pack's) voltage drops slightly. This reliability is the same as C1.ps (also C2.ss, C3ps, C4.ss, C5.ps, C6.ss, C7.ps, and C8.ss in Table IV), and C2.s for the cell-level (C4.s, C6.s, and C8.s for the array-level). When they get stuck-at OFF state, the S-switch becomes critical, resulting in the same reliability as in the case of B-switches. The reliability of every configuration is presented in Table IV.

Since a switch's life varies with the current load imposed on it, the mean lifetime of each switch $\left(\lambda_{\mathrm{SW}}^{*}\right)$ is determined using fractions of the charge current load shown in Tables II and III with a normalization factor $\tau$ and an exponential random variable $\lambda_{\text {SW }}$ defined. The mean life for the main switch used in each configuration is also listed in Table IV.

\section{EVALUATION}

To evaluate the dependability and scalability of DESA, we use metrics that include the power dissipation on switches, the 
TABLE V

PARAMETERS AND VALUES

\begin{tabular}{|c|c|c|}
\hline Parameter & Description & Value \\
\hline \hline$F$ & Fault coverage & 0.05 \\
\hline$\lambda_{a}$ & Average \# of defects & 3 \\
\hline$\alpha$ & Clustering & 2 \\
\hline$C_{A}$ & Manfg. cost per array (normalized) & 1 \\
\hline$N$ & \# of arrays & {$[20,40]$} \\
\hline$M$ & Min. \# of available arrays & {$[10,20]$} \\
\hline$C_{F}$ & Service cost per array & $3 \times C_{A}$ \\
\hline$n$ & \# of cells per array & {$[15,20]$} \\
\hline$m$ & Min. \# of available cells per array & {$[15,20]$} \\
\hline $1 / \lambda_{B}$ & battery-cell lifetime (years) & 23 \\
\hline $1 / \lambda_{S}$ & 3-Ampere switch lifetime (years) & $1.5 / \lambda_{B}$ \\
\hline$p$ & Prob. of bypassing an array & 0.05 \\
\hline$q$ & Prob. of bypassing a cell & 0.05 \\
\hline$r$ & \# of arrays connected to load & {$[7,10]$} \\
\hline$s$ & \# of cells connected to load & {$[15,20]$} \\
\hline$x$ & Current Ampere (coulombs) & {$[1,10]$} \\
\hline$\tau$ & Normalization factor & $2\left(C^{*}+C^{+}\right)$ \\
\hline
\end{tabular}

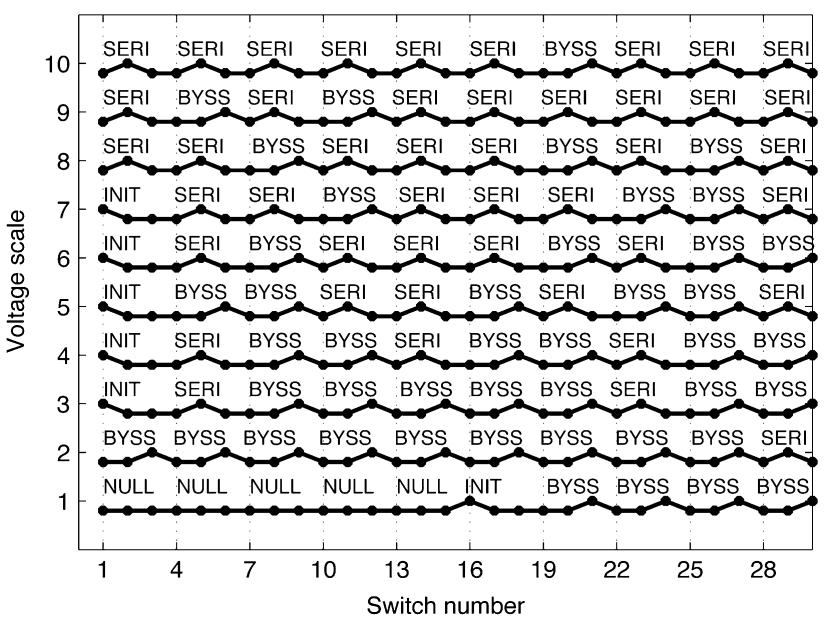

Fig. 5. Switching command array: The array of switches is systematically configured depending on required voltages.

reliability of the entire battery system, and the service cost associated with failures. Also, the parameters presented in Section V are listed and specified in Table V.

In what follows, using the above metrics and parameters, we demonstrate DESA's superiority to a conventional BMS without reconfigurability.

A Sequence of Command Codes Matches A Switch Array: Five command codes are defined to turn on/off switches. In the cell-level arrangement, the local (global) controller first determines which battery-cells (arrays) are to be turned on. After this determination with the barr parameter set, the local (global) controller applies the algorithm in Fig. 2, forming a combination of on- and off-switches. Fig. 5 shows the correspondence between a sequence of command codes and the combination of switches. Without loss of generality, we assume that every battery-cell's (array's) voltage is $1 \mathrm{~V}$. For instance, when setting the total voltage to 1 , the local controller turns on Switch 16, resulting in Cell 6 (i.e., the 7th cell) to be active. Cells before (after) Cell 6 become open (bypassed).

To build a $2 \mathrm{~V}$ array, Cells 0 and 10 are connected in series. In general, the INIT code indicates the beginning of an active battery-cell array. That is, the preceding battery cells are ignored in

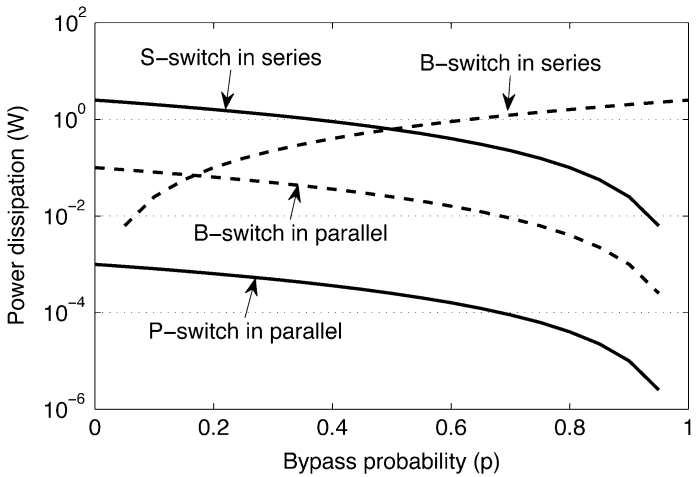

Fig. 6. Power dissipation on each switch in series and parallel arrangements.

spite of their connectivity. Cell-bypassing is applied effectively when the switches associated with the preceding battery cells are dysfunctional. This feature minimizes the impact of failure of a single battery cell or switch that might otherwise cause the entire battery-cell array to fail.

Power Dissipation Varies With the Type of Switch: Three types of switch are used in DESA: P-, S-, and B-switches. Each switch incurs power dissipation because of their unavoidable internal resistance. Their power dissipation differs in parallel or series arrangement. In the series arrangement, the S-switch is turned on. As shown in Fig. 6, it consumes a significant amount of power. In contrast, to bypass a battery cell, its B- and S-switches are turned on and off, respectively. Thus, the likelihood of bypassing a battery cell dictates the power dissipation on the switches; a higher bypass-probability results in lower power dissipation on the corresponding S-switch and higher power dissipation on the corresponding B-switch. In the parallel arrangement, the P-switch is turned on. The power dissipation thereon, however, is negligible compared to the $\mathrm{S}$-switch in the series arrangement, since the current to the load is shared across the battery cells. By contrast, the B-switch in parallel serves as the conductor for the parallel-connected battery-cells. Its power dissipation, thus, depends solely on the distributed current. In general, it is two orders-of-magnitude higher than that on the $\mathrm{P}$-switch. Note that the $\mathrm{S}$-switch is never turned on in the parallel arrangement.

Redundancy Greatly Improves the Battery Pack'S Reliability: The battery pack operates as long as at least $M$-out-of- $N$ battery-cell arrays function. Likewise, the array operates as long as at least $m$-out-of- $n$ cells function. In other words, $(n-m) M+$ $(N-M) n$ battery cells can be used as backups. The more the backup cells available, the longer the battery pack will last. DESA is more effective than the conventional BMS in utilizing redundant battery cells. As shown in Fig. 7(a), DESA improves the pack's reliability an average of $2.7 \times$ over the conventional BMS. Moreover, DESA can effectively handle a large number of battery cells. As shown in Fig. 7(c), DESA improves the reliability $3.5 \times$ with a tenfold increase in the number of available battery cells in an array $(m)$, compared to the conventional BMS, which cannot deal with a large number of battery cells.

Desa Allows the Battery Pack to Last Longer: Although individual battery cells last long, e.g., an average of 23 years, the lifetime of the battery pack formed by these cells is not guaranteed to last that long. Actually, it is subject to the arrangement of 


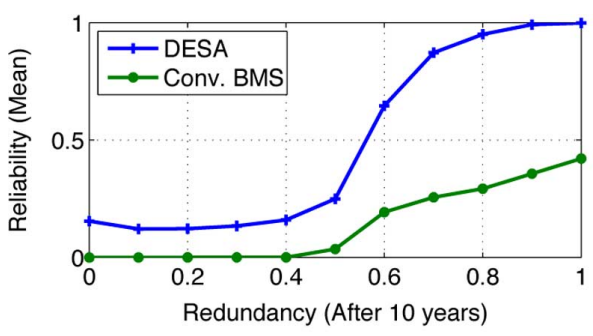

(a)

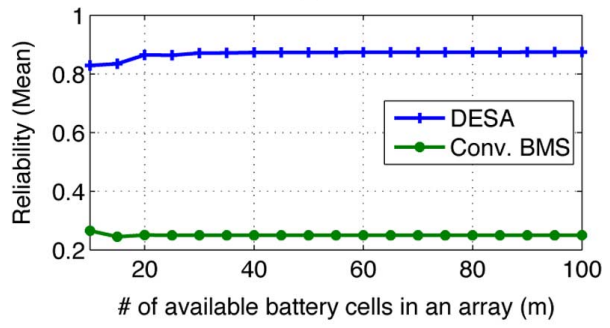

(b)

Fig. 7. Reliability versus redundancy $\left(\left(1 / \lambda_{B}\right)=23,\left(1 / \lambda_{\mathrm{SW}}\right)=34, t=\right.$ 10). (a) Mean redundancy ( $m=20, M=10)$. (b) Array size at the redundancy rate of 0.7 .

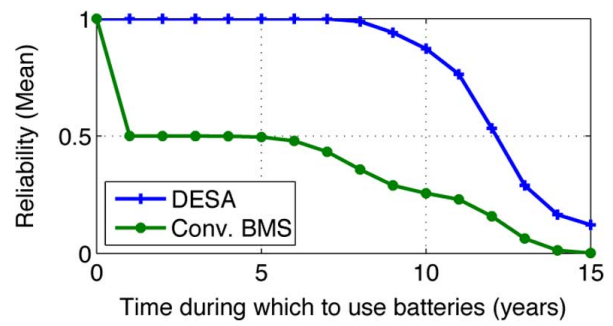

(a)

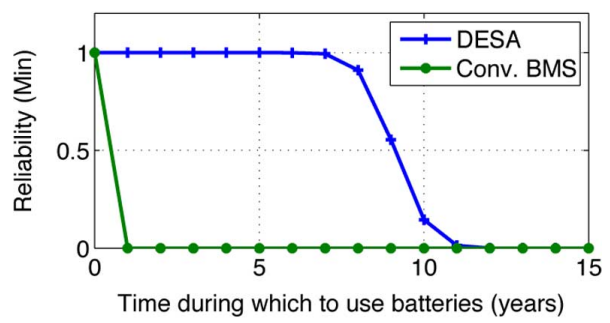

(b)

Fig. 8. Change in reliability with the pack used at the redundancy rate of 0.7. (a) Mean reliability. (b) Minimum reliability.

cells and their (random) failures. ${ }^{2}$ For instance, when the battery cells are connected in parallel, a short-circuited cell causes the entire battery pack to be unusable. In the case of series arrangement, an open-circuited cell has the same consequence. DESA effectively prevents such a single cell failure from causing the entire pack to fail. As shown in Fig. 8(a), on average, DESA offers twice as much reliability as the conventional BMS over the battery's lifetime. In particular, a DESA-managed battery pack to be used for ten years is $3 \times$ more reliable than the conventional BMS-managed one, whose reliability is only $26 \%$. In the worst-case scenarios such as those mentioned earlier, as shown in Fig. 8(b), DESA is an order-of-magnitude more reliable than the conventional BMS, which is susceptible to the loss of the entire pack due to the failure of a single battery cell. Thus, DESA

${ }^{2}$ The more cells in the system, the more likely some of them may fail.

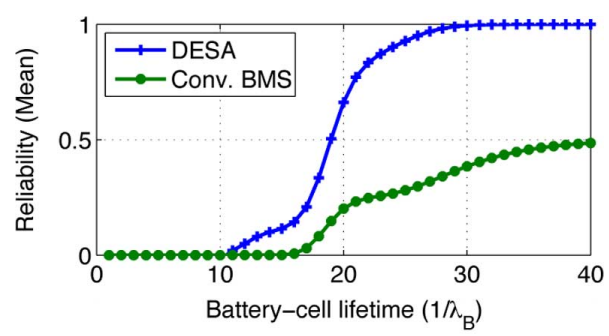

(a)

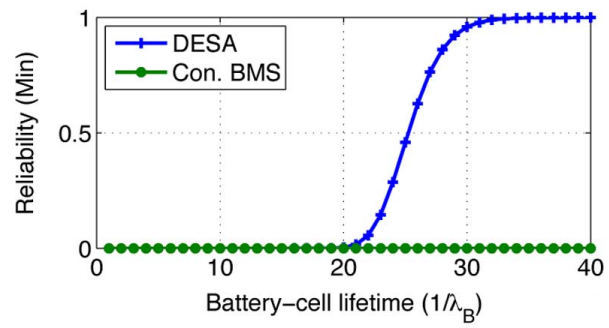

(b)

Fig. 9. The battery pack's reliability with respect to a battery-cell lifetime at the redundancy rate of 0.7 . (a) Mean reliability. (b) Minimum reliability.

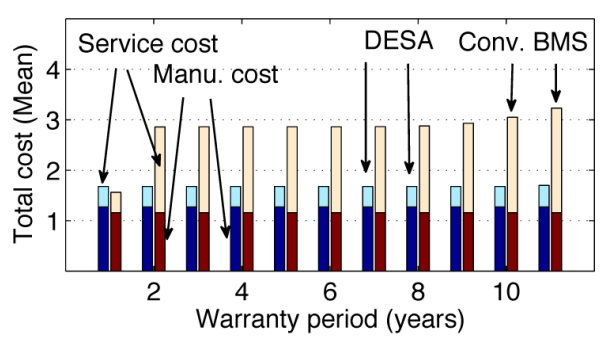

(a)

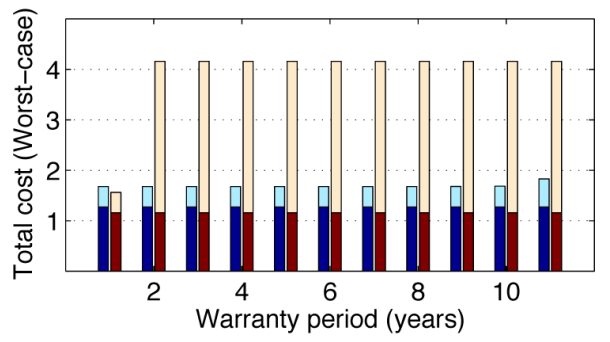

(b)

Fig. 10. Change in the total cost for a single battery pack with respect to warranty periods. (a) Average total cost. (b) Total cost in the worst case.

offers robust battery management regardless of the type of failures that might occur.

DESA Can Always Offer a Dependable and Affordable Life Warranty of Battery Packs: What types of battery cell do we need to meet the requirement that a battery pack must last for ten years with 50\% reliability? Fig. 9 answers this question. DESA requires battery cells of 20-year life warranty, while the conventional BMS requires those of 40-year life warranty. Theoretically, a DESA-managed battery pack would be manufactured twice more affordably than a conventional BMS-managed one. In the worst-case scenarios, as shown in Fig. 9(b), the conventional BMS cannot provide any warranty for the battery pack, whereas DESA requires battery cells of 26-year life to meet the requirement. 
Desa Enables the Production of Cost-Effective Battery Packs: DESA requires switches around each cell, increasing the manufacturing cost. However, since a set of switches costs much less than battery cells, the manufacturing cost for DESA is not much different from that for the conventional BMS, as shown in Fig. 10(a). Rather, the service cost, which is associated with the reliability, has a great impact on the total cost. It is important to reduce the service cost because it is usually much costlier $($ e.g., $3 \times$ ) than the manufacturing cost. DESA reduces the service cost by an average of $4.2 \times$ compared to the conventional BMS over various warranty periods. In the worst-case scenarios, DESA makes more than $7 \times$ of cost-savings for a longer than ten-year warranty period. As a result, DESA makes battery packs affordable and dependable.

\section{CONCLUSION}

In this paper, we have presented a combined hardware-software architecture, called DESA, that enables effective monitoring of a large number of battery cells, and efficient control and reconfiguration of their connection arrangement. In DESA, the global BMS orchestrates as many local BMSs - that autonomously manage their battery-cell arrays and switches-as required. DESA allows individual local BMSs and their arrays to be selectively turned off, making significant energy-savings. This savings has been verified by our evaluation based on a Markov model. Also, DESA's systematic switch configuration algorithm allows the battery connection arrangement to be dynamically changed, and hence, any faulty cells to be effectively bypassed. By effectively tolerating failures of battery cells and switches, DESA extends the battery life significantly compared to the conventional BMS. Moreover, it yields more than $7 \times$ cost-savings for a longer than 10-year warranty period, making battery packs more affordable and attractive. Integration of the switch configuration algorithm and the reconfigurable architecture enhances the reliability, customizability, and extensibility of large-scale battery packs.

\section{REFERENCES}

[1] C. Blake and C. Bull, "Igbt or Mosfet: Choose Wisely." [Online]. Available: http://www.irf.com/technical-info/papers.html

[2] Bosch. Can specification version 2. [Online]. Available: http://www. semiconductors.bosch.de/pdf/can2spec.pdf, 1991

[3] Bosch, "Road vehicles-Controller area network (CAN)-Part 2: High-speed medium access unit," Tech. Rep. 11898-2, ISO, 2003.

[4] Bosch, "Road vehicles-Controller area network (CAN)-Part 3: Low-speed, fault-tolerant, medium-dependent interface," Tech. Rep., 11898-3, ISO, 2006.

[5] S. Ci, J. Zhang, H. Sharif, and M. Alahmad, "A novel design of adaptive reconfigurable multicell battery for power-aware embedded network sensing systems," in Proc. IEEE Globecom, Washington, DC, 2007, pp. 1043-1047.

[6] Green Car Congress. "Noaa: February 2009 ninth warmest February for globe." [Online]. Available: http://www.greencarcongress.com/2009/03/noaa-february-2.html, Mar. 2009

[7] A. Davis, Z. M. Salameh, and S. S. Eaves, "Evaluation of lithium-ion synergetic battery pack as battery charger," Trans. Energy Conversion, vol. 14, no. 3, pp. 830-835, 1999.

[8] J. T. de Sousa and V. D. Agrawal, "Reducing the complexity of defect level modeling using the clustering effect," in DATE. New York: ACM, 2000, pp. 640-644.

[9] Infineon, Linear Hall Sensor.

[10] H. Kim and K. G. Shin, "On dynamic reconfiguration of a large-scale battery system," in Proc. IEEE RTAS, San Francisco, CA, Apr. 2009, pp. 87-96.
[11] H. Kim and K. G. Shin, "Scheduling of battery charge, discharge, and rest," in Real-Time Systems Symp., Washington, DC, Dec. 2009, pp. 13-22, IEEE Computer Society.

[12] J. Markoff, "Nyt: Pursuing a battery so electric vehicles can go the extra miles." [Online]. Available: http://www.nytimes.com/2009/09/15/science/15batt.html?scp=1\&sq=electric vehicle tesla $20 \mathrm{ibm} \& \mathrm{st}=\mathrm{cse}$ Sep. 2009

[13] MAXIM. Temperature sensors. [Online]. Available: http://www. maxim-ic.com/pl_list.cfm/filter/11

[14] D. McNamara, "Thermal sensing requires system-level design," Power Electron. Technol., pp. 28-35, May 2007.

[15] General Motors, "Latest Chevy volt battery pack and generator details and clarifications." [Online]. Available: http://gm-volt. com/2007/08/29/latest-chevy-volt-battery-pack-and-genera tor-details-and-clarifications/ Aug. 2007

[16] S. Shamshiri, P. Lisherness, S.-J. Pan, and K.-T. Cheng, "A cost analysis framework for multi-core systems with spares," in Proc. IEEE Int. Test Conf., New York, 2008, pp. 1-8.

[17] T. Stuart, F. Fang, X. Wang, C. Ashitiani, and A. Pesaran, "A modular battery management system for HEVs," in Proc. SAE Int., Hyatt Crystal City, VA, Jun. 2002, pp. 1-9,

[18] V. Sukumar, M. Alahmad, K. Buck, H. Hess, H. Li, D. Cox, F. N. Zghoul, J. Jackson, S. Terry, B. Blalock, M. M. Mojarradi, W. C. West, and J. F. Whitacre, "Switch array system for thin film lithium microbatteries," J. Power Sources, vol. 136, no. 2, pp. 401-407, 2004.

[19] H. Visairo and P. Kumar, "A reconfigurable battery pack for improving power conversion efficiency in portable devices," in Proc. IEEE Int. Caribbean Conf. Devices, Circuits and Systems (ICCDCS), Apr. 2008, pp. 1-6.

[20] C. Yang, D. McCollum, R. McCarthy, and W. Leighty, "Meeting an $80 \%$ reduction in greenhouse gas emissions from transportation by 2050: A case study in California," Transp. Res. Part D: Transport and Environment, vol. 14, no. 3, pp. 147-156, 2009.

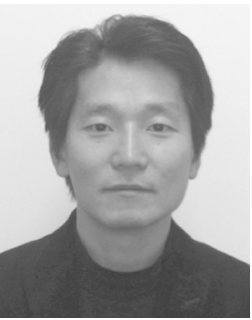

Hahnsang Kim (M'09) received the B.S. and M.S. degrees in computer science and engineering from Korea University, Seoul, Korea, in 1998 and 2000, respectively, and the Ph.D. degree in computer science from the Institut National des Télécommunications (INT), France, in 2006.

$\mathrm{He}$ is a Senior Research Fellow of the Real-Time Computing Laboratory, Department of Electrical Engineering and Computer Science, University of Michigan, Ann Arbor, since 2007. From 2000 to 2005, he was a member of the Planète Team at the Institut National de Recherche en Informatique et en Automatique (INRIA) Sophia Antipolis, France, the Expert Engineer from 2000 to 2003, and then a Research Assistant until 2005. He was also affiliated with the Department of Computer Science, Imperial College London, U.K., in 2006. His current research focuses on embedded real-time and cyberphysical systems, with emphasis on security and dependability.

Dr. Kim is a corecipient of the Plug-in Hybrid Electric Vehicle Translational Grant (2009), the Engineering Translational Research Award (2011), and the Michigan Universities Commercialization Initiative Award (2011). He is a coauthor of the NSF EAGER Grant 2011 named SmartGreen.

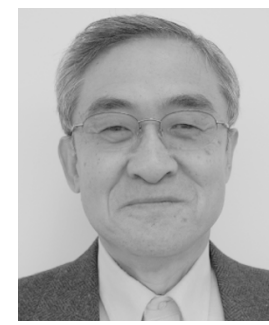

Kang G. Shin (F'92) received the B.S. degree in electronics engineering from Seoul National University, Seoul, Korea, in 1970, and the M.S. and $\mathrm{Ph} . \mathrm{D}$. degrees in electrical engineering from Cornell University, Ithaca, NY, in 1976 and 1978, respectively.

$\mathrm{He}$ is the Kevin and Nancy O'Connor Professor of Computer Science and Founding Director of the Real-Time Computing Laboratory, Department of Electrical Engineering and Computer Science, University of Michigan, Ann Arbor. From 1978 to 1982 he was on the faculty of Rensselaer Polytechnic Institute, Troy, NY. He also chaired the Computer Science and Engineering Division, Department of Electrical Engineering and Computer Science, University of Michigan for three years beginning January 1991. He has supervised the completion of $65 \mathrm{Ph} . \mathrm{D} . \mathrm{s}$, authored/coauthored more than 720 technical articles and more than 20 patents. He has coauthored (with C. M. Krishna) a textbook "Real-Time Systems," (McGraw-Hill, 1997). His current research focuses on computing systems and networks as well as on embedded real-time and cyberphysical systems, all with emphasis on timeliness, security, and dependability. 